lassen. Es ist bislang nicht deutlich geworden, ob die hier in sehr eingeschränkter Weise ermöglichte erleichterte Einbürgerung tatsächlich zu einer spürbaren Veränderung des Einbürgerungsverhaltens der betreffenden Angehörigen der zweiten und dritten Generation geführt hat. Eine der Hauptschwierigkeiten ist nach wie vor das Problem der doppelten Staatsangehörigkeit. Gerade männliche Jugendliche werden aus ihrer bisherigen Staatsangehörigkeit nur dann entlassen, wenn sie ihren Militärdienst absolviert oder sich mit einer entsprechend hohen Summe davon freigekauft haben (wie vor allem in den Fällen der türkischen Staatsangehörigen). Für eine erleichterte Einbürgerung sind deshalb - wie von der Bundesbeauftragten für Ausländerfragen wiederholt zur Diskussion gestellt - wirksame Maßnahmen erforderlich, die vor allem das Problem der Mehrfachstaatsangehörigkeit zum Gegenstand haben. ${ }^{4}$

Klaus Sieveking

\title{
Rassismus als Problem des Völkerrechts
}

Rassismus oder genauer Rassendiskriminierung stellt sich für das Völkerrecht in zwei Formen dar, die wohl zu unterscheiden sind: einmal als Diskriminierung bestimmter Bevölkerungsgruppen, zum anderen als Diskriminierung einzelner Mitglieder wegen der Zugehörigkeit zu dieser Gruppe. Auf die erste Form antwortet das Völkerrecht durch Minderheitenschutzsysteme, wie wir sie aus der Völkerbundzeit kennen. In völkerrechtlichen d.h. zwischenstaatlichen Verträgen bilateraler oder multilateraler Art verpflichten sich Staaten, Teilen ihrer Staatsanghörigen, die sich nach bestimmten Merkmalen z. B. religiöser, nationaler bzw. ethnischer Art von anderen unterscheiden, einen Sonderstatus, meist auf kulturellem Gebiet (Sprache, Schule etc.) einzuräumen. Auf dieses Problem, das seit einiger Zeit in den Vordergrund gerückt ist (die laufende Vollversammlung diskutiert eine UN-Deklaration zum Minderheitenschutz, demnächst soll ein Entwurf des Schutzes von sog. indigenous peoples behandelt werden), kann ich aus Zeitgründen nicht einghen. Ich will aber zumindest darauf hinweisen, daß die Illusion von 1945, Minderheitenprobleme seien historisch überholt, längst zerstoben ist und das Völkerrecht der gesellschaftlichen und internationalen Problementwicklung folgen muß.

Auf die zweite Form der Rassendiskriminierung antwortet das Völkerrecht durch Einräumung von Menschenrechten und deren Garantie für alle Menschen ohne Unterschiede, die ebenfalls in völkerrechtlichen Verträgen zwischen den Staaten vereinbart werden. Prominentes Beispiel ist Art. 27 des IPBürgR:

$\leadsto$ In Staaten mit ethnischen, religiösen oder sprachlichen Minderheiten darf Angehörigen solcher Minderheitn nicht das Recht vorenthalten werden, gemeinsam mit anderen Angehörigen ihrer Gruppe ihr eigenes kulturelles Leben zu pflegen, ihre eigene Religion zu bekennen um auszuüben oder sich ihrer eigenen Sprache zu bedienen."

Hier wird ein individuelles Recht eingeräumt, das allerdings nur existieren kann, wenn die Gruppe als solche in ihrer Existenz gesichert ist. Indirekt ist also ein Minderheitenschutz vorausgesetzt. Umstritten ist, ob sich diese Verpflichtung nur auf eigene Staatsangehörige des betreffenden Staates bezieht oder auch auf Ausländer auf dessen Territorium, somit auch auf Arbeitsimmigranten.

\footnotetext{
4 Im März 1993 sind von den Bundestagsfraktionen CDU/CSU, SPD und F.D.P. sowie von der Ausländerbeauftragten der Bundesregierung Gesetzesvorschläge zur Neuordnung des Staatsangehórigkeitsrechts vorgelegt worden, die auf eine vermehrte Hinnahme der Doppelstaatsangehörigkeit abziehen.
} 
$\mathrm{Da}$ nach 1945 das Problem der Rassendiskriminierung völkerrechtlich nur als Menschenrechtsproblem betrachtet wurde, ist auf zwei Gründe zurückzuführen. Zum einen die mißbräuchliche Benutzung der Minderheitenschutzverträge der Völkerbundzeit, insbesondere durch das Deutsche Reich und Nazideutschland.

Stichwort: Polen, Sudetenland, wo die deutschen Minderheiten als sog. 5. Kolonne benutzt wurde und der Minderheitenschutz später in ein rassistisches Volksgruppenrecht pervertierte. Der andere Grund besteht drin, daß man, wie schon erwähnt, nach den fürchterlichen Genoziden und Bevölkerungsverschiebungn zischen 1939 und I945 glaubte, das Problem nationaler Minderheiten sei historisch erledigt. Die Folge jedenfalls war, daß im Kontext der Vereinten Nationen die Rassendiskriminieung bis in die jüngste Zeit vornehmlich als individuelles Menschenrechtsproblem behandelt wurde. Hintergrund für alle Regelungen war die schwerwiegende, massenhafte und systematisch betriebene Menschenrechtsverneinung des deutschen Faschismus. Das reicht von der UN-Charta, die völkerstrafrechtlichen Versuche des Nürnberger Gerichtshofes, die Normierung der Menschenrechte insbesondere in den beiden Pakten von I 966 bis zu den großen Konventionen über die sog. Rassendiskriminierung und die Sanktionierung des Apartheidverbrechens. Im folgenden beschränke ich mich auf die Frage der Rassendiskriminierung.

Ausgangspunkt jeder völkerrechtlichen Überlegung heute ist das »Internationale Übereinkommen zur Beseitigung jeder Form von Rassendiskriminierung v. 7. 3. $1966 \ll$, das die Bundesrepublik Deutschland 1969 ratifiziert hat. Bemerkenswert in der Präambel der Konvention ist der Hinweis, daß »jede Lehre von einer auf Rasseunterschieden gegründeten Überlegenheit wissenschaftlich falsch, moralisch verwerflich sowie sozial ungerecht und gefährlich ist « und daß die Rassendiskriminierung geeignet ist, »den Frieden und die Sicherheit unter den Völkern wie das harmonische Zusammenleben der Menschen sogar innerhalb eines Staates zu stören«.

Nach Art. 1 ist Rassendiskriminierung »jede auf Rasse, der Hautfarbe, der Abstammung, dem nationalen Ursprung oder dem Volkstum beruhende Unterscheidung, Ausschließung, Beschränkung oder Bevorzugung « mit der Zielsetzung oder der Folge, Menschenrechte aller Kategorien zu beeinträchtigen. Unterscheidungen, die an der Staatsangehörigkeit anknüpfen, werden allerdings ausdrücklich ausgenommen.

Art. 1 Abs. 3 lautet: „Dieses Übereinkommen ist nicht so auszulegen, als berühre es die Rechtsvorschriften der Vertragsstaaten über Staatsangehörigkeit, Staatsbürgerschaft oder Einbürgerung, sofern diese Vorschriften nicht Angehörige eines bestimmten Staates diskriminieren. «

In Bezug auf Ausländer geht die Regelung offensichtlich vom gewohnheitsrechtlichen Mindeststandard der Menschenrechte aus. Im darüber liegenden Standard müssen aber alle Ausländer gleichbehandelt werden. An diesem Kriterium gemessen wäre z. B. die Unterscheidung zwischen Ausländern aus verfolgungsfreien und verfolgenden Staaten problematisch, wie sie in die Asylrechtsdiskussion eingeführt wurde.

Art. 2 enthält die grundsätzliche Verpflichtung der Staaten, nicht nur rassendiskriminierende Handlungen zu unterlassen, also im Verhältnis der Staatsgewalt zum Bürger. Vielmehr verpflichten sich die Vertragsstaaten, »... mit allen geeigneten Mitteln unverzüglich eine Politik der Beseitigung der Rassendiskriminierung in jeder Form ... zu verfolgen: zu diesem Zweck ... verbietet und beendigt jeder Vertragsstaat jede durch Personen,Gruppen oder Organisationen ausgeübte Rassendiskriminierung mit allen geeigneten Mitteln einschließlich der durch die Umstände erforderlichen Rechtsvorschriften.«

Art. 4 und 5 präzisieren einerseits alle Bereiche, die historisch besonders durch Rassendiskriminierung betroffen waren. Hier wird der ganze Katalog der Menschenrechte aufgeführt, der in die Pakte von 1966 aufgenommen wurde.

Gemäß Art. 4 übenehmen die Staaten die Verpflichtung, »... jede Verbreitung von Ideen, die sich auf die Überlegenheit einer Rasse oder den Rassenhaß gründen, jedes Aufreizen zur Rassendiskriminierung und jede Gewalttätigkeit oder Aufreizung dazu gegen eine Rasse oder Personengruppe anderer Hautfarbe oder Volkszugehörigkeit sowie jede Unterstützung rassenkämpferischer Betätigung zu einer nach dem Gesetz strafbaren Handlung zu erklären."

Anders als andere Vertragsstaaten hat die Bundesrepublik darauf verzichtet, besondere gesetzliche Bestimmungen für die nationale Implementierung dieser Bestim- 
mungen zu schaffen. Mit Blick auf Art. 3 Abs. 3 GG, $\mathbb{\$} 130$ StGB sowie die zur Verfügung stehenden Normen des öffentlichen und des Privatrechts wurde dies für überflüssig gehalten, anders ausgedrückt, es wird behauptet (so die Bundesregierung), der normative Level des Übereinkommens sei schon längst erreicht. Das kann bezweifelt werden. Manche Gerichte verurteilten zwar gemäß $\$ S$ I 30, I 3 I StGB antisemitische Agitation durch Broschüren, das öffentliche Leugnen der Judenvernichtung, die Identifizierung mit nationalsozialistischen Verbrechern oder die pauschale und öffentliche Hetze und Verächtlichmachung von Schwarzen. Andere Gerichte hingegen sahen Probleme. Der BGH verlangt z. B., daß der Angriff gegen den unverzichtbaren und unableitbaren Persönlichkeitskern des anderen, gegen sein Menschsein als solches gerichtet ist und ihm diesen Weert abspricht. Der im Tatbestand enthaltene Begriff der Menschenwürde wird nicht nur als Einschränkung der Strafbarkeit verstanden, sondern zudem noch restriktiv ausgelegt. So wird im Lokal-

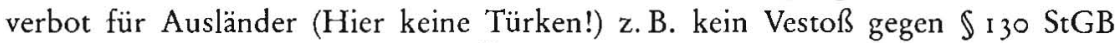
gesehen, obwohl Art. se und $f$ des Übereinkommens gegen Rassendiskriminierung Verpflichtungen aufstellen, die für Private gelten, z. B. »das Recht auf Zugang zu jedem Ort oder Dienst, der für die Benutzung durch die Öffentlichkeit vorgesehen ist«, wie Verkehrsmittel, Hotels, Gaststätten, Cafés, Theater, Parks.

Es ist mehr als fraglich ob diese Praxis mit der Konvention übereinstimmt. Das kam auch vor dem Ausschuß zur Beseitigung der Rassendiskriminierung (Internationale Kontrolle) zur Sprache, als der Bericht der Bundesregierung von 1982 diskutiert wurde. Es wurde von Mitgliedern des Ausschusses vorgetragen, der Tatbestand des $\$$ I 30 StGB müßte enger gefaßt werden. Der Vertreter der Bundesregierung allerdings meinte, eine völkerrechtsfreundliche Auslegung würde genügen. Das mag zutreffen. Nur folgen die Gerichte dieser Empfehlung nicht, so daß der Gestzgeber gefordert wäre.

Im Kontext dieser internationalen Kritik kam immerhin I $985 \$ 131$ neu in das StGB. Er erfaßt Handlungen, die von $\$ I 30 StGB nicht oder nur teilweise abgedeckt werden. Danach wird die Verbreitung von Schriften, die zum Rassenhaß aufstacheln, unter Strafe gestellt. In ihrem Bericht von 1986 weist die Bundesregierung auf diese Änderung hin. Dennoch hat sich die Rechtsprechung der Strafgerichte hinsichtlich des Lokalverbotes für Ausländer, bislang nicht verändert. Auch die Verwaltungspraxis hat z.B. den Entzug der Gaststättenerlaubnis wegen Unzuverlässigkeit des Gewerbetreibenden nach $\rrbracket_{4}$ Abs. I GastG bzw. $\$ 35$ Abs. I GewO noch nicht erwogen, obwohl er in der Literatur im Zusammenhang mit einer bestimmten Auslegung des Begriffs der öffentlichen Ordnung vorgeschlagen wird.

Ähnliches wurde in der zivilrechtlichen Diskussion in Bezug auf wertausfüllungsbedürftigen Generalklauseln (gute Sitten etc.) vorgeschlagen. Immerhin normiert der Gesetzgeber in $\$ \$ 75,80$ Betriebsverfassungsgesetz, daß Arbeitgeber und Betriebsrat darüber zu wachen haben, daß keine unterschiedliche Behandlung der im Betrieb tätigen Personen wegen ihrer Abstammung oder Nationalität erfolgt.

Kurzum: Es ist fraglich, ob die Bundesrepublik ihren Verpflichtungen aus dem Übereinkommen gegen Rassendiskriminierung nachkommt. Wenn schon immer nach dem Gesetzgeber gerufen wird - meist wird nur die Verstärkung dubioser Instrumente: Nachrichtendienste, Kronzeugenregelung etc. gefordert -, dann wäre eine Präzisierung der Strafrechtsnormen bzw. der unbestimmten Rechtsbegriffe des Verwaltungsrechts entsprechend dem Übereinkommen bzw. anderer menschenrechtlicher Bestimmungen (Pakte) ein wichtiges und sinnvolles Betätigungsfeld des Gesetzgebers.

Gerhard Stuby 